\title{
Hydrodynamic (HD) Simulation of $N$-Channel MOSFET's with a Computationally Efficient Inversion Layer Quantization Model
}

\author{
HAIHONG WANG*, WEI-KAI SHIH, SUSAN GREEN, SCOTT HARELAND, \\ CHRISTINE M. MAZIAR and AL. F. TASCH JR.
}

Microelectronics Research Center, The University of Texas at Austin, Austin, TX 78712, USA

\begin{abstract}
A quantum mechanical treatment of electron inversion layers is incorporated in the hydrodynamic (HD) transport model used in UT-MiniMOS. A physically based, yet computationally efficient, three-subband model is implemented in the HD simulation tool. The three-subband model, which is based upon solutions to Schrodinger's equation, has the important advantage of more accurately predicting the distribution of electrons in the inversion layers than does more conventional classical models. A more simplified quantum mechanical model with carrier heating effects included has also been developed. Terminal currents are calculated using these quantum mechanical models and the comparison with results from classical calculations indicates the importance of quantum mechanical effects in the deep submicron device simulations.
\end{abstract}

Keywords: MOS, inversion layer, quantization, hydrodynamic simulation, carrier confinement

\section{INTRODUCTION}

The quantum mechanical treatment of carrier confinement in silicon MOSFET inversion layers is increasingly essential for accurate simulation of devices scaled into the deep submicron region. It is well known that a high transverse electric field at the $\mathrm{Si} / \mathrm{SiO}_{2}$ interface leads to a large energy band bending, which in turn can cause quantization of the inversion layer carriers in the direction per- pendicular to the interface [1]. Self-consistent solutions of the Schrödinger's and Poisson equations have been adopted by a number of researchers to investigate the two-dimensional nature of electrons in inversion layers $[2,3]$. However, from the point of view of the device design engineer, less computationally expensive approaches that may be routinely used with existing device simulators are necessary. Van Dort's simple quantum model [4] and three subband quantization model (3SB) [5]

\footnotetext{
* Corresponding author
} 
have been implemented in drift-diffusion simulation tools and have facilitated the prediction of device electrical behavior (such as threshold voltage and capacitance).

The three subband model introduces an additional level of physical sophistication by solving for the energy-level and wave-function for the first three subbands, using approximate solutions to Schrödinger's equation based upon physically reasonable estimates of the form of the potential well. This approach has the advantage of correctly predicting the electron distribution in the inversion layers due to quantum mechanical effects and, in turn, leads to better predictive capabilities for C$\mathrm{V}$ simulation and physical oxide thickness estimation. In the hydrodynamic (HD) 3-subband simulation, carrier temperature instead of lattice temperature is used to distribute electrons among each subband and between the classical (three dimensional electron gas) and the quantum (two dimensional electron gas) domains. As a result, under high drain bias condition, a large portion of electrons near the drain edge will be in the classical domain due to the carrier heating effect. In this paper, we present, for the first time, HD simulation results with a $3 \mathrm{SB}$ model included using UTMiniMOS [6].

\section{CARRIER CONFINEMENT AND QUANTIZATION MODEL IMPLEMENTATION}

When carriers are quantized in the potential well, the density of states must be represented by a twodimensional (2-D) density of states instead of the three-dimensional (3-D) density of states that is assumed in classical calculations. Knowing the eigenvalue and eigenfunction for each subband, we can represent the distribution of charge in a 2-D system by the following expression:

$$
n_{\mathrm{QM}}(z)=\frac{k T_{\text {carrier }}}{\pi \hbar^{2}} \sum_{i} n_{v i} m_{z i}
$$

$$
\sum_{j} \ln \frac{\left\{1+\exp \left(\frac{\left(E_{F}-E_{i j}\right)}{k T_{\text {carrier }}}\right)\right\}}{\left\{1+\exp \left(\frac{\left(E_{F}-E_{\mathrm{cl}}\right)}{k T_{\text {carrier }}}\right)\right\}}\left|\zeta_{i j}(z)\right|^{2}
$$

where $n_{v i}$ denotes the degeneracy of the $i^{\text {th }}$ subband, $E_{i j}$ the subband energy level of the $j^{\text {th }}$ valley in the $i^{\text {th }}$ subband, $E_{\mathrm{cl}}$ the energy level of the classical domain, and $\zeta_{i j}(z)$ the wavefunction describing the distribution of carriers in the subband. In the three subband model, $E_{i j}$ and $\zeta_{i j}$ are calculated via self-consistent solutions of Schrödinger's equation and Poisson's equation. However, a priori assumptions about the shape of the potential well are used to solve (1) based upon analytical expressions for $E_{i j}$ and $\zeta_{i j}$. A triangular potential well is used in the three subband model. For this form of the potential, the solution to Schrödinger's equation is the Airy function where the eigenenergies for the system can be calculated exactly as a function of the surface field $[2,5]$. To account for the perturbation of the potential due to the inversion layer charges, a variational wavefunction originally proposed by Fang and Howard [7] was used for the lowest subband. This bypasses the computational expense of solving Schrödinger's equation explicitly. In order to maintain additional computational efficiency, only the first three subbands are used in the calculation of (1). Above the energy level $E_{\mathrm{cl}}$, the carriers are all treated as classical carriers with a 3-D density of states. This approach ensures agreement with classical calculations in the limit of low channel doping in addition to reducing the total computation time.

Since the electron temperature (or average energy) is used as an additional variable in the HD simulation, the temperature effect must be included in the quasi-Fermi level calculation in order to obtain a correct carrier distribution among subbands. Also, due to the electron heating, the classical carrier component may play an important role in the carrier density calculation for the region near the drain edge. The quasi- 
Fermi level and the classical carrier component need to be calculated correctly in the 3SB model to ensure that the HD simulation converges and that the simulation results are physically correct. In our approach, the fractional electron population in the quantum domain as a function of the electron energy is modeled using our quantum-window enhanced uniform-field Monte Carlo (MC) simulator [8], as shown in Figure 1. The fractional population-energy relationship in our HD simulation is obtained from Monte Carlo simulation under the low transverse effective field condition $\left(E_{\text {trans }}=1.9 \times 10^{5} \mathrm{~V} / \mathrm{cm}\right)$. Very little impact on the terminal current, even under the high drain bias condition, is observed by choosing a different fractional population-energy relationship from Monte Carlo simulations with different transverse effective fields. The reasons for the insensibility of terminal current on the fractional populationenergy relationship are being under investigations. The fractional population, so modeled, can then be used to calculate the sheet quantum carrier concentration $\left(N_{\mathrm{qm}}\right)$ and classical carrier concentration $\left(N_{\mathrm{cl}}\right)$ for a given total sheet carrier concentration $\left(N_{\mathrm{qm}}+N_{\mathrm{cl}}\right)$ after the drift-diffusion solution is obtained. By knowing $N_{\mathrm{qm}}$ and $N_{\mathrm{cl}}$, we can calculate the quasi-Fermi level and classical

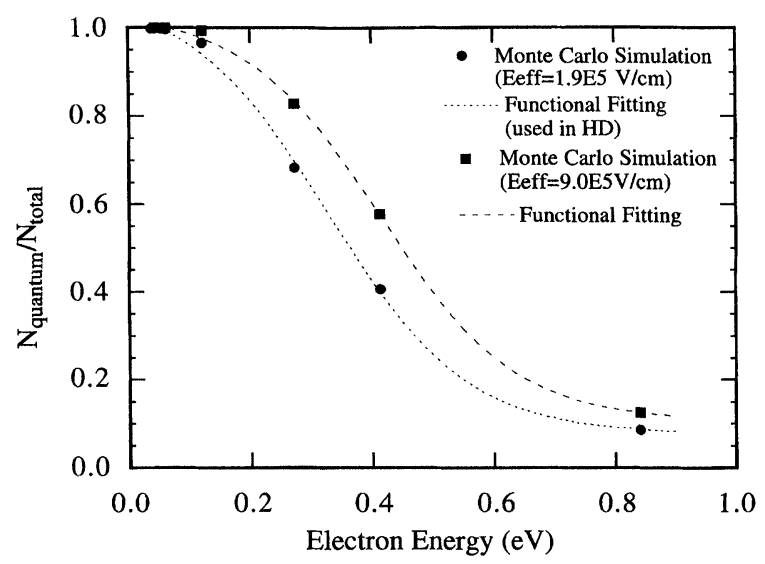

FIGURE 1 Fractional population of electrons in the quantum domain (first three subbands) as a function of the electron average energy from our uniform-field Monte Carlo simulation. A simple analytic fit used in the hydrodynamic simulation is also plotted. effective density of states. The carrier distribution along the depth direction is subsequently calculated in the 3SB model to obtain the effective intrinsic carrier density, following the similar approach of Ref. [5].

In addition to the more physically accurate three-subband model, more simplified models are of interest to the modeling community. While these models do not attempt to predict the quantum mechanically consistent charge distribution, they are able to predict the effect of quantization on the terminal characteristics when this is the only information required. This is accomplished by altering the bandgap of the silicon near the interface via the intrinsic carrier concentration [4]. The change in the bandgap is modeled as follows:

$$
\Delta E_{G}=\beta\left(\frac{\varepsilon_{\mathrm{si}}}{4 q k T_{\text {carrier }}}\right) E_{s}^{2 / 3}
$$

where $E_{s}$ denotes the transverse electric field and $\beta$ the fitting parameter used to calibrate the model to experimental data and/or more physically based quantization models. This modified bandgap is then used to make corrections to the charge density near the interface. The carrier temperature is used when the HD models are turned on, otherwise the lattice temperature is used. This modification permits the model to approach the classical results when the energy distribution of the carriers is highly energetic, and hence more classical-like.

\section{SIMULATION RESULTS AND DISCUSSION}

In order to explore the importance of including carrier confinement models, as well as carrier heating models, in device simulation tools, we have examined the behavior of the drift-diffusion and hydrodynamic transport models both with quantum mechanical carrier confinement models and without. We used as our prototypical device structure the simple 0.18 micron nMOS device 
presented in Figure 2. The doping profiles, junctions depths and oxide thickness were selected to be representative of devices of this technology generation.

An examination of the electron concentration near drain edge at low drain bias $\left(V_{d}=0.1 \mathrm{~V}\right)$ illustrates that the electron distribution obtained from the HD 3SB calculation is almost the same as that from a DD-3SB calculation (Fig. 3(a)). This result can be understood by the fact that there is almost no carrier heating for such a low drain bias. At high drain bias $\left(V_{d}=1.8 \mathrm{~V}\right)$, on the other hand, the distribution near the drain edge obtained from the HD- 3SB calculation differs significantly from that obtained with DD 3SB (Fig. 3(b)). In the drift-diffusion approach, regardless of lateral field conditions, the carriers are assumed to be at the lattice temperature, so the carriers are assumed to occupy the lowest subbands (i.e., quantum mechanical regime) more readily, and hence have a smaller classical contribution (i.e., the unbound states). However, in the hydrodynamic approach, under high lateral electric field conditions, significant carrier heating can occur near the drain edge. Such energetic carriers tend to occupy bound states in the higher subbands and the unbound
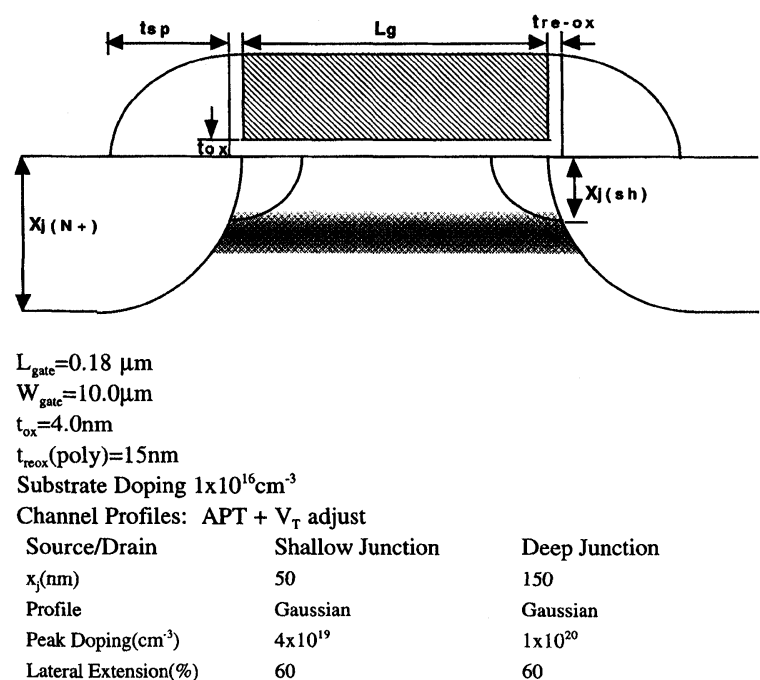

FIGURE 2 Device structure for prototypical 0.18 micron nMOS transistor. This structure is used throughout the results section of the paper.
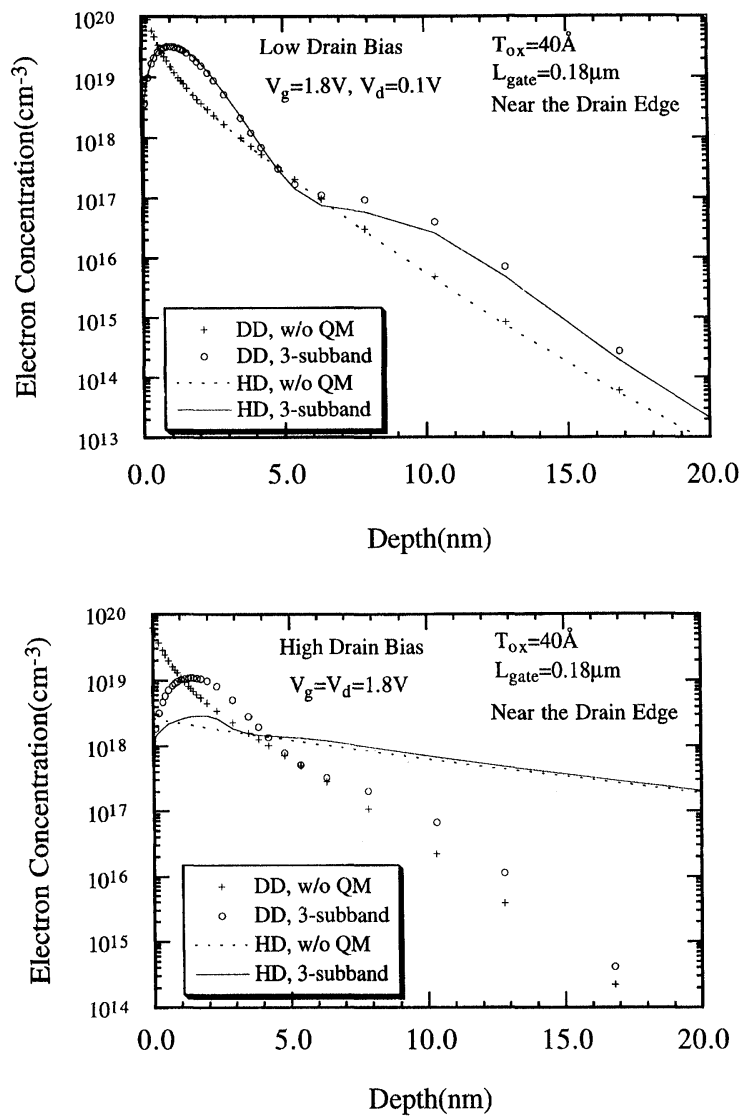

FIGURE 3 (a) Electron concentration along the depth near the drain edge at the low drain bias $\left(V_{d}=0.1 \mathrm{~V}\right)$ for both DD and HD simulations (classical and 3-subband) (b) Electron concentration along the depth near the drain edge at the high drain bias $\left(V_{d}=1.8 \mathrm{~V}\right)$ for both DD and HD simulations (classical and 3-subband).

states. Hence, for high drain bias, the electron distribution near the drain edge calculated from HD 3-subband is more classical-like.

The $I_{d}-V_{d}$ characteristics from the HD simulations are presented in Figure 4. Either a temperature corrected van Dort's model or the 3-subband quantum mechanical model is used in the HD quantum mechanical calculations. The results from classical HD simulation are also presented for comparison. It should be noted that the drain currents from the temperature corrected van Dort's model agree very well with those from the three subband HD simulations. Also, the observable difference in saturation currents between 


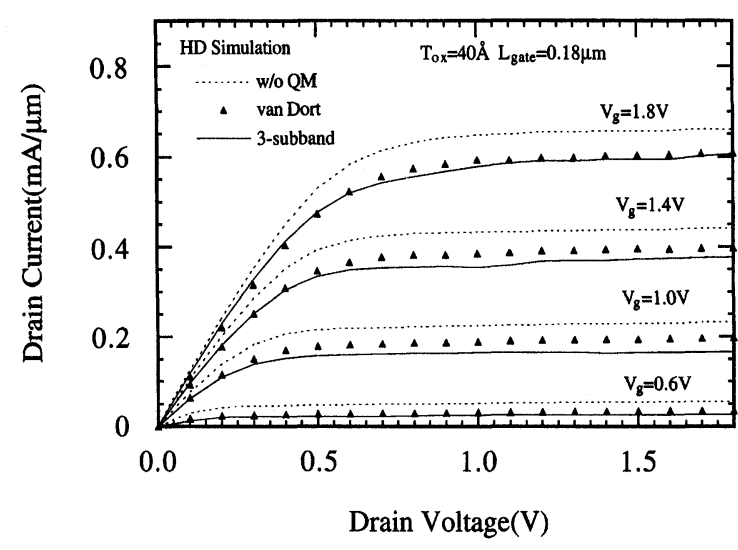

FIGURE 4 Drain currents versus drain voltage from the hydrodynamic simulation, using classical, temperature corrected van Dort's model and 3-subband model, respectively.

classical and quantum mechanical calculation suggests that the carrier confinement effects should be taken into account for the deep submicron device simulation. The $I_{d}-V_{g}$ characteristics from HD simulation with classical, temperature corrected van Dort model, and 3-subband model, respectively (Fig. 5), indicate that the two quantum models predict almost the same threshold voltage, while the threshold voltage from classical HD calculations is smaller than that from quantum mechanical HD calculations. The difference in the threshold voltage between the classical and quantum mechanical calculations is believed to

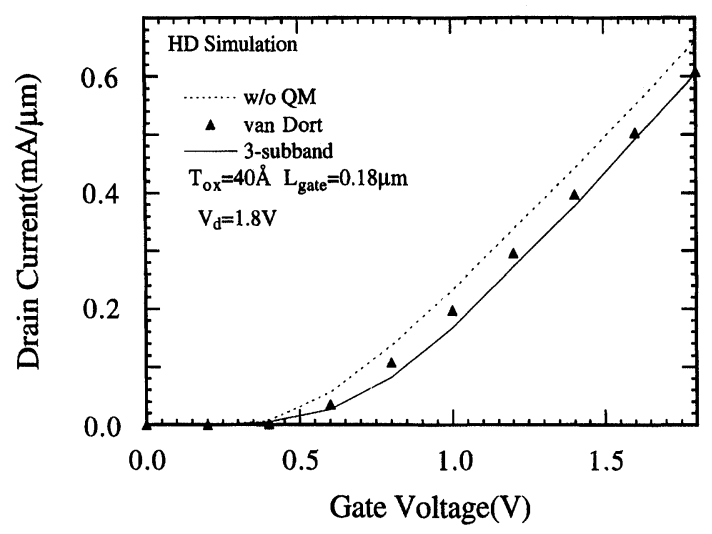

FIGURE 5 Drain current versus gate voltage from the hydrodynamic simulation, using classical, temperature corrected van Dort's model and the 3-subband model, respectively. contribute to the difference in the saturation drain current between HD classical and HD quantum mechanical calculations, as shown in Figure 4.

\section{CONCLUSION}

Carrier confinement and quantization models have been successfully implemented in the hydrodynamic (HD) simulation tool UT-MiniMOS. Under high drain bias conditions, the electron concentration near the drain edge calculated from HD simulation differs significantly from that of DD simulation due to the carrier heating effect, both with and without quantum effects included. Under low dran bias conditions, quantum confinement effects are significant while carrier heating effects are negligible. The $I_{d}-V_{d}$ and $I_{d}-V_{g}$ characteristics suggest the need for both the HD treatment and the QM picture either through the use of the 3SB model or a temperature corrected van Dort model.

\section{Acknowledgements}

This work was supported, in part by the Semiconductor Research Corporation (SRC), the Joint Services Electronics Program (F49620-95-C-0045), the Texas Advanced Technology Program (TATP) and Motorola. The United States Government is authorized to distribute reprints for governmental purposes notwithstanding any copyright notation hereon.

\section{References}

[1] Schriffer, J. R. (1957). "Mobility in Inversion Layers: Theory and Experiment", in Semiconductor Surface Physics, p. 55.

[2] Stern, F. (1972). "Self-Consistent Results for $n$-type Si Inversion Layers", Phys. Rev. B, 5(12), 4891.

[3] Ando, T., Fowler, A. B. and Stern, F. (1982). "Electronic Properties of Two-Dimensional System", Rev. Mod. Phys., 54(2), 437.

[4] van Dort, M. J., Woerlee, P. H. and Walker, A. J. (1994). "A Simple Model for Quantization Effects in HeavilyDoped Silicon MOSFETs at Inversion Conditions", SolidState Electron., 37(3), 411. 
[5] Hareland, S. A., Krishnamurthy, S., Jallepalli, S., Yeap, C.-F., Hasnat, K., Tasch, A. and Maziar, C. (1996). "Computationally Efficient Models for Inversion Layer Quantization Effects in Deep Submicron $N$-Channel MOSFETs", IEEE Trans. Electron Devices, 43(1), 90.

[6] UT-MiniMOS 5.2-3.2 User's Guide, Microelectronics Research Center, The University of Texas at Austin, 1997.

[7] Fang, F. F. and Howard, W. E. (1966). "Negative FieldEffect Mobility on (100) Si Surface", Phys. Rev. Lett., 16(8), p. 797.

[8] Wei-Kai Shih, Scott Hareland, Srinivas Jallepalli, Maziar, Christine M. and Tasch, Al. F. (1996). "An Accurate Preprocessor for Monte Carlo Study of Electron Transport in Inversion Layers of Silicon nMOSFETs", 54th Annual Device Research Conference Digest, p. 28.

\section{Authors' Biographies}

Haihong Wang received the B.S. degree in physics from Beijing University, Beijing, P.R.C., in 1988, and the M.S. degree in electrical engineering from the University of Texas in Austin in 1995. He is currently pursuing the Ph.D. degree in electrical engineering at the University of Texas at Austin. His research interests include advanced transport models and quantum-mechanical effects in deep submicron MOSFET's, low-power device design and analysis, and improved structures for MOSFET scaling.

Wei-Kai Shih received the B.S. degree in physics from National Taiwan University, Taipei, Taiwan, R.O.C., in 1989, and the M.A. degree in physics from the University of Texas at Austin in 1994. He is currently pursuing the Ph.D. degree in electrical engineering at the University of Texas at Austin. His research interests include hot-carrier effects, non-local phenomena, and quantum-mechanical effects in deep submicron MOSFET's.

Susan Green received the B.S.E.E. degree from the University of Arizona in 1994 and the M.S.E. from the University of Texas at Austin in 1996. She is currently with Intel in Chandler, Arizona.
Scott Hareland received the B.S.E.E. degree from Rice University in 1992 and the M.S.E. and Ph.D. degrees from the University of Texas at Austin in 1994 and 1997. He is currently with Intel in Portland, Oregon. His research interests include device, process and circuit modeling, device physics, and improved structures for MOSFET scaling. Dr. Hareland was awarded an Intel Foundation Robert Noyce Memorial Fellowship in 1994 and is a member of Phi Beta Kappa, Tau Beta Pi and Eta Kappa Nu.

Christine Maziar received the B.S.E.E. (with Highest Distinction), the M.S.E.E. and the Ph.D. degrees from Purdue University in 1981, 1984, and 1986. In January of 1987, she joined the faculty of the University of Texas at Austin, where she is a Professor of Electrical and Computer Engineering and Vice Provost. She holds the Archie W. Straition Endowed Teaching Fellowship in Electrical and Computer Engineering. Her research interests include modeling of charge transport in high performance device structures, semiconductor device physics, and device simulator enhancement. She has authored or co-authored over 100 journal or conference publications. She is a member of IEEE, ASEE, APS, Eta Kappa Nu, Tau Beta Pi and Sigma Pi Sigma.

AL. F. Tasch received his Ph.D. degree in Physics in 1969 form the University of Illinois, Urbana-Champaign. He worked in industry for 17 years at Texas Instruments and Motorola, and joined the faculty of the Department of Electrical and Computer Engineering at the University of Texas at Austin in 1986 as a Chair Professor. He has been awarded 38 U.S. patents and was elected to the National Academy of Engineering in 1989. 

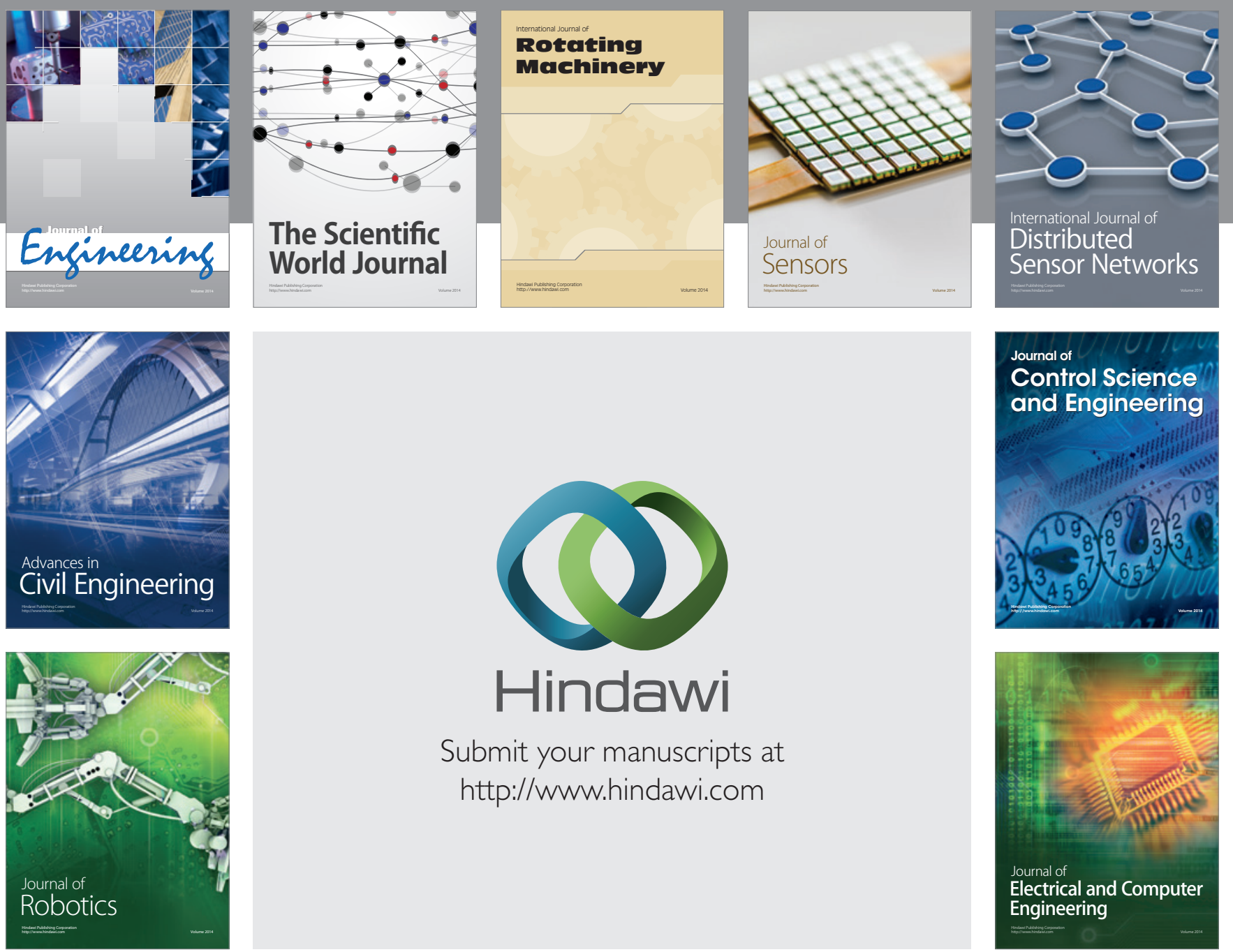

Submit your manuscripts at

http://www.hindawi.com
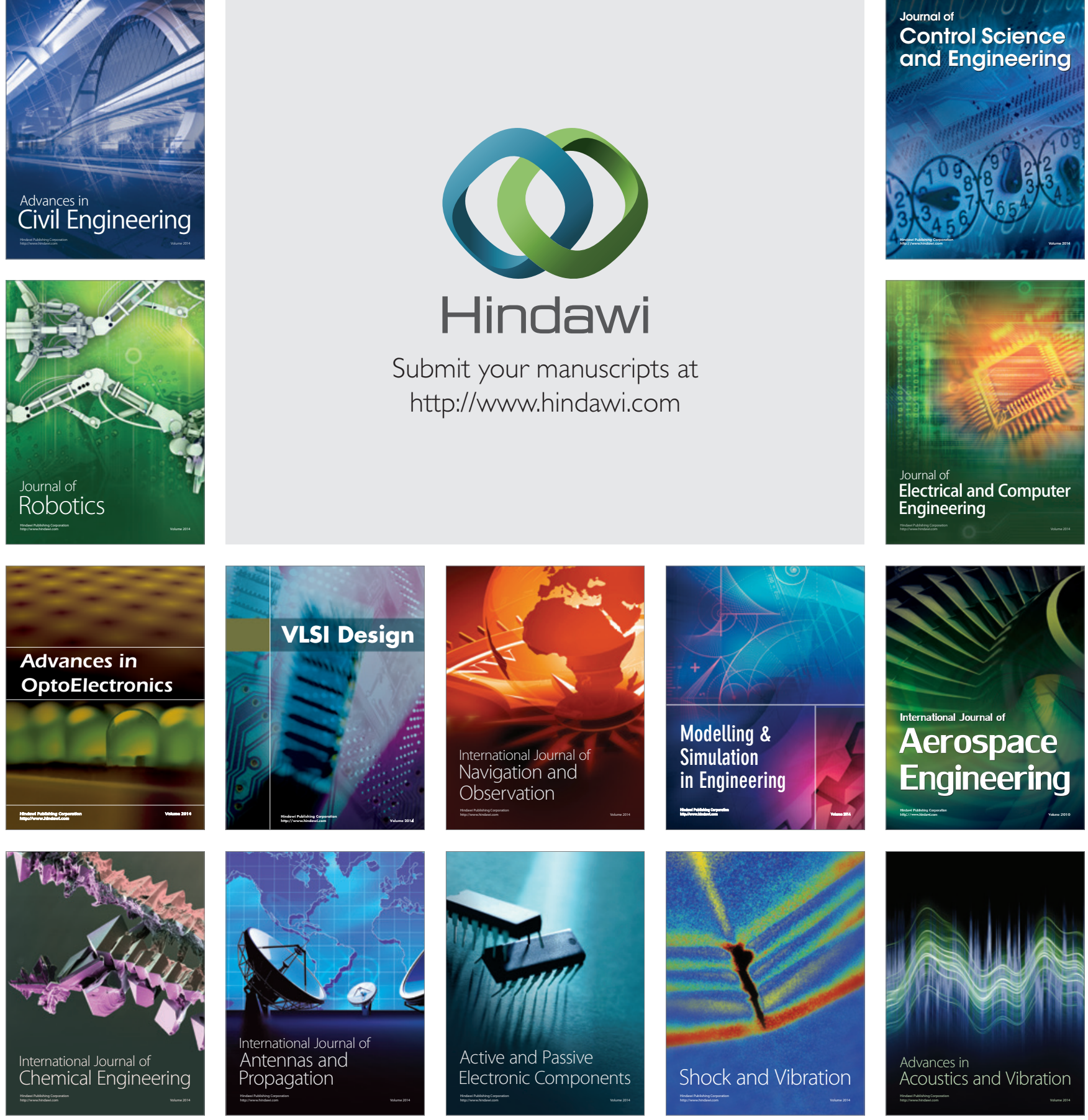